\title{
INVESTIGACIONES
}

\section{Políticas educativas y currículo en la emergencia sanitaria de 2020}

\author{
Educational policies and curriculum in the 2020 health emergency \\ Políticas educacionais e currículo na emergência sanitária de 2020
}

\author{
María Sol Villagómez R. ${ }^{a}$, Daniel Llanos Erazo ${ }^{a}$ \\ ${ }^{a}$ Universidad Politécnica Salesiana del Ecuador. \\ mvillagomez@ups.edu.ec,dllanos@ups.edu.ec
}

\begin{abstract}
RESUMEN
La rapidez de los acontecimientos y el crecimiento exponencial de los casos de contagio por Covid-19 en el mundo obligó a los Estados a tomar decisiones sobre la marcha. Desde una perspectiva tradicional e incluso genérica, asumimos que dicha toma de decisiones por el gobierno de un Estado es comprendida como política pública o por lo menos, lineamientos de política. Varios de estos lineamientos responden a lógicas instrumentales que están preocupadas por las metodologías y procedimientos para [...] solucionar un problema o conflicto emergente o presente en la sociedad (Pulido, 2017, p. 16). Por ello, el presente artículo pretende analizar las políticas educativas ecuatorianas y el currículo en el marco de la emergencia sanitaria por la pandemia provocada por el Covid-19. En el componente metodológico, optamos por enfoques mixtos buscando una triangulación metodológica que combinó el análisis documental, ciberetnografía y encuesta, acudiendo así a métodos cuantitativos, en el caso de la encuesta y cualitativos en el caso de la ciberetnografía que se realizó a través de apoyos virtuales. Entre los principales resultados identificamos que las políticas educativas implementadas por el gobierno ecuatoriano no consideraron los accesos tecnológicos y de conectividad de docentes y estudiantes, lo que provocó una situación de desasosiego en estos dos actores del sistema educativo. Asimismo, y a partir de la triangulación metodológica identificamos que el Plan Covid 19 fue subutilizado por los docentes en sus actividades áulicas. Las reflexiones finales nos invitan a cavilar acerca de la necesidad del dialogo constate. Las decisiones educativas, sobre el diseño y desarrollo curricular deberán ser siempre consensuadas con los diferentes actores de la comunidad educativa.
\end{abstract}

Palabras claves: Políticas educativas, políticas curriculares, práctica docente, emergencia sanitaria.

\begin{abstract}
The rush of events and the exponential growth of cases of contagion by Covid-19 in the world forced States to make decisions on the fly. From a traditional and even generic perspective, we assume that such decisionmaking by the government of a State is understood as public policy or at least, policy guidelines. Several of these guidelines respond to instrumental logics that are concerned with the methodologies and procedures to [...] solve an emerging or present problem or conflict in society (Pulido, 2017, p. 16). Therefore, this article aims to analyze Ecuadorian educational policies and the curriculum in the framework of the health emergency caused by the Covid-19 pandemic. In the methodological component, we opted for mixed approaches looking for a methodological triangulation that combined documentary analysis, cybernetnography and survey, thus resorting to quantitative methods, in the case of the survey, and qualitative methods in the case of cybernetnography, which was carried out through support virtual. Among the main results, we identify that the educational policies implemented by the Ecuadorian government did not consider the technological and connectivity accesses of teachers and students, which caused a situation of unease in these two actors of the educational system. Likewise, and from the methodological triangulation, we identify that the Covid 19 Plan was underused by teachers in their
\end{abstract}


classroom activities. The final reflections invite us to ponder about the need for constant dialogue. Educational decisions on the design and development of the curriculum must always be agreed with the different actors of the educational community.

Key words: Educational policies, curricular policies, teaching practice, health emergency.

\section{RESUMO}

A rapidez dos eventos e o crescimento exponencial dos casos de contágio da Covid-19 no mundo forçaram os Estados a tomar decisões rapidamente. De uma perspectiva tradicional e até genérica, partimos do pressuposto de que tal tomada de decisão por parte do governo de um Estado é entendida como política pública ou, pelo menos, diretrizes de política. Várias dessas diretrizes respondem a lógicas instrumentais que se preocupam com as metodologias e procedimentos para [...] resolver um problema ou conflito emergente ou presente na sociedade (Pulido, 2017, p. 16). Portanto, este artigo tem como objetivo analisar as políticas educacionais equatorianas e o currículo no marco da emergência sanitária causada pela pandemia Covid-19. Na componente metodológica, optamos por abordagens mistas buscando uma triangulação metodológica que combinasse análise documental, cibernografia e survey, recorrendo a métodos quantitativos, no caso do survey, e métodos qualitativos, no caso da cibernetnografia, que foi realizada em suporte virtual. Dentre os principais resultados, identificamos que as políticas educacionais implementadas pelo governo equatoriano não consideraram os acessos tecnológicos e de conectividade de professores e alunos, o que gerou um mal-estar nesses dois atores do sistema educacional. Da mesma forma, e a partir da triangulação metodológica, identificamos que o Plano Covid 19 foi subutilizado pelos professores em suas atividades em sala de aula. As reflexões finais nos convidam a refletir sobre a necessidade de um diálogo constante. As decisões educacionais sobre a concepção e desenvolvimento do currículo devem ser sempre acordadas com os diferentes atores da comunidade educacional.

Palavras-chave: Políticas educacionais, políticas curriculares, prática docente, emergência sanitária.

\section{INTRODUCCIÓN}

La rapidez de los acontecimientos y el crecimiento exponencial de los casos de contagio por Covid-19 en el mundo obligó a los Estados a tomar decisiones sobre la marcha. Desde una perspectiva tradicional e incluso genérica, asumimos que dicha toma de decisiones por el gobierno de un Estado es comprendida como política pública o por lo menos, lineamientos de política. Por lo general, y como una respuesta propia de gobiernos anclados a lógicas pragmáticas, la política pública se aborda desde visiones técnicas e instrumentales preocupadas por las metodologías y procedimientos para [...] solucionar un problema o conflicto emergente o presente en la sociedad (Pulido, 2017, p. 16). Esta noción instrumental de la política es propia de modelos neoliberales que predominan en varios gobiernos del mundo y por supuesto de nuestra región.

En lo que respecta a la definición de política pública, es preciso mencionar que los aportes desde la ciencia política y de la sociología política son predominantes en la literatura especializada. Los recambios ideológicos que han caracterizado a los regímenes gubernamentales de América Latina en los últimos 15 años se incrementó la reflexión acerca de la importancia de las políticas públicas centradas en los gobiernos de turno y no en los recetarios provenientes de "tecnócratas-intelectuales" de diferentes organismos de cooperación. A pesar de estos giros políticos producidos en los países del cono sur y de la zona andina de América, el enfoque instrumental y otros desde una perspectiva crítica, a la cual suscribimos son los que se encuentran en la palestra del debate académico.

En ese sentido, siguiendo una línea crítica y centrada en la necesidad de la presencia de un Estado fuerte y organizador de la vida social, consideramos que la política pública 
es "el proceso por el cual se elaboran y se implementan programas de acción pública, es decir dispositivos político-administrativo coordinados, en principio, alrededor de objetivos explícitos" (Muller y Surel, citados en Roth, 2009, p. 26) que procuran atender de forma efectiva las problemáticas sociales.

Esta idea procesual de la política es el resultado de un diagnóstico de la problemática identificada a solucionar por parte de los administradores gubernamentales, sin embargo, la valoración no siempre es realizada, y, por el contrario, la linealidad casi mecánica, expresada en la identificación del problema -sin un diagnóstico- conlleva a una búsqueda y propuesta de soluciones que no siempre resuelven la dificultad establecida. Este actuar político gubernamental, se basa en preceptos de optimización de recursos y tiempos que a la larga terminan siendo todo lo contrario, porque los problemas no fueron resueltos y las "medidas de acción" generaron nuevas problemáticas.

Esta noción de política instrumental, que atiende las problemáticas de forma casi "intuitiva" y sin mayor profundización analítica de las causas generadoras, también se encuentra presente en las políticas educativas. Este enfoque de corte neoliberal, a nuestro entender, puede generar niveles de opacidad y encubrimiento de las problemáticas. En ese sentido, y siguiendo las recomendaciones instrumentales de la formulación de políticas, estos lineamientos se caracterizan por ser sectoriales, instalando la narrativa gubernamental de eficiencia y eficacia gracias al cruce sectorial, es decir, el principio de políticas intersectoriales se convierte en la regla a seguir.

Si bien en la teoría la idea de intersectorialidad se constituye como la alternativa más viable que facilita el proceso de implementación de las políticas públicas, en la realidad este tipo de decisiones no siempre son las acertadas, a saber, que las problemáticas "entrañan" orígenes propios de un sector social y que no necesariamente pueden ser resueltos de forma intersectorial. Por ello, es preciso apostar por la planificación y organización de las acciones a seguir. Ciertamente, esta planificación debe realizarse en el espacio que ostenta el poder; "porque la política y lo político es inmanente al poder, hasta tal punto que para que se dé la política debe haber poder efectivo" (Capella, J., s/f., s. p.).

La pandemia actual puso a prueba la capacidad organizativa y de respuesta de todos los países del mundo. La necesidad de atender a la población en su conjunto se tradujo en la toma de decisiones urgentes y carentes de diagnósticos. Dichas decisiones fueron difundidas sin mayor consideración de la realidad social de cada país, en varias naciones se instalaban "acciones replicas" de lo ya realizado en otras latitudes. En el caso particular de Ecuador, el gobierno del presidente Moreno siguió esta corriente y tomó decisiones símiles a las ya impuestas en Europa, algunas sin duda, con el vértigo propio de la ausencia de planificación gubernamental.

Las políticas educativas para el caso del sistema educativo ecuatoriano siguen el orden de lo antes expuesto la primera disposición del Ministerio de Educación fue la suspensión de las actividades escolares en los centros de estudios de todos los niveles. Esta decisión se tomó el miércoles 11 de marzo como una acción para precautelar la salud de niñas/os y adolescentes y entró en vigencia a partir del viernes 13. Tres días después de la suspensión de actividades académicas, es decir, el 14 de marzo, la ministra del ramo, presenta al país 'El Plan Covid-19'. Este documento contiene los lineamientos generales para que docentes, directivos, Departamentos de Consejería Estudiantil -DECE- y las Unidades Distritales de Apoyo a la Inclusión-UDAI-, continúen con las actividades pedagógicas mientras dura la emergencia sanitaria declarada por el gobierno central. 
Este plan tiene como objetivo continuar las actividades académicas de los educandos desde sus hogares. El documento contempla varias acciones didácticas que los docentes deben trabajar bajo la modalidad a distancia. En el documento también se enfatiza la obligatoriedad de los centros educativos de programar tutorías abiertas y opcionales, a partir del uso de los recursos digitales que se pusieron a disposición en la plataforma ${ }^{1}$ diseñada por el Ministerio.

Es importante señalar que las decisiones y acciones emergentes en el campo educativo fueron pensadas, desde las instancias ministeriales, por ello, toda acción originada o sugerida desde un espacio de gobierno, se constituye como ya lo mencionamos líneas arriba, en una política, a saber, que los lineamientos políticos procuran y se encamina a solucionar o prevenir una situación definida como problemática (Velásquez Gavilanes, 2009, p. 156). En este caso, la inactividad educativa que se producía por el confinamiento era el problema a solucionar.

En esa dirección, el Ministerio de Educación optó por la implementación de un modelo de teleducación temporal, es decir, las declaraciones de los funcionarios gubernamentales apuntaban a la suspensión de clases por tiempos acotados y, por lo tanto, los niños/as y adolescentes debían realizar actividades pedagógicas en casa, previendo que, al retorno a las actividades presenciales, las tareas serían evaluadas y validadas por los docentes. Esta idea de un pronto retorno a la modalidad presencial generó confusiones y desorientaciones en el grupo de docentes, padres de familia y por supuesto, en los mismos estudiantes.

El desconcierto social se profundizó cuando la cartera de educación anunció que las medidas de suspensión de actividades escolares permanecerán por tiempo indefinido. En el mismo comunicado se explicó que "desde el lunes 23 de marzo se empezaría a difundir programas educativos en 160 canales de televisión, cableoperadoras y en 1.000 radios rurales-comunitarias".

Este anuncio que tenía como finalidades el salvaguardar la salud de la población estudiantil y continuar con el proceso educativo, provocó incomodidades en la ciudadanía porque los programas, obviamente, no se encontraban listos y formateados para cumplir fines pedagógicos.

Unos días más tarde el Ministerio de Educación anunció que se implementará estrategias de teleducación en todas las instituciones educativas del país. Esta estrategia buscaba garantizar el aprendizaje diario de los estudiantes y el bienestar de los miembros de la comunidad educativa.

La ministra de la cartera de educación aseguraba en cadenas nacionales, notas de prensa y a través de los portales institucionales que "el aprendizaje mediante una plataforma educativa virtual ofrece ventajas en cuanto al entorno de la flexibilidad de horarios, mayor diversidad de recursos y la posibilidad de colaborar e intercambiar experiencias en el desarrollo de competencias digitales" (Ministerio de Educación, 2020). Estas decisiones exacerbaron, aún más, los ánimos de la población ecuatoriana, sobre todo de aquellos sectores que habitan en zonas rurales del país y carecen de conectividad. Un claro ejemplo de la improvisación y ausencia de diagnósticos a la hora de implementar políticas educativas.

La posibilidad de acceso y conectividad a redes de internet en las zonas rurales del país es mínima. Algunos datos extra oficiales señalan que para el 20 de marzo de este año

Plataforma Aprendamos juntos en casa https://bit.ly/3ebU2Yw 
se comprobó que solo el $37 \%$ de hogares tiene acceso a internet (16\% en la zona rural). De esos $37 \%$ del total de hogares, solo el $24 \%$ tiene computadoras en casa ( $8 \%$ en la zona rural) (Oviedo, 2020). Con esa realidad, en lo que respecta a la accesibilidad de servicios y recursos tecnológicos la situación educativa en el Ecuador se tornó más compleja.

Las brechas de desigualdad social y educativa, presentes antes de la pandemia, se visibilizan con nitidez en la población estudiantil y docente. Más de un docente expuso en medios de comunicación social su preocupación por no contar con internet en casa o equipos tecnológicos que faciliten la labor educativa. Si a eso le sumamos que la capacitación en manejo de recursos tecnológicos -plataformas- es ausente en el quehacer educativo, podemos afirmar que la educación durante los primeros 90 días de la pandemia entró en una suerte de estado cataléptico.

Las decisiones curriculares vienen de la mano con las políticas hasta aquí expuestas, así al inicio de la pandemia se experimentó una suerte de medidas de corto plazo para las 6630 unidades educativas que forman parte del ciclo sierra del país. Estas medidas luego fueron modificándose con el avance de la pandemia y la obligatoriedad que los 215.255 docentes del sistema educativo nacional mantengan el teletrabajo en este marco se diseña el Plan educativo Covid-19 que a decir de la instancia reguladora consistía en un "currículo compactado"; "esto significaba orientarnos mucho en trabajar la parte emocional y la realización de una valoración del portafolio de cada uno de los alumnos" (Ministerio de Educación del Ecuador, 2020), teniendo como objetivo "que los estudiantes continúen con las actividades académicas desde sus hogares".

Bajo este mismo esquema se preveía el inicio del año escolar en el ciclo costa y Galápagos. El currículo y la oferta educativa priorizaría tres aspectos fundamentales "la atención inmediata a la situación emocional de las familias, el desarrollo de contenidos pertinentes a la realidad y la aplicación de un modelo de evaluación a través del diseño de portafolios" (Ministerio de Educación, 2020). Lamentablemente, como se ha expuesto previamente, las condiciones contextuales, económicas y de conectividad, no permiten que los estudiantes de todo el sistema educativo avancen en este acometido.

Durante la emergencia sanitaria, el diseño curricular fue experimentando varias modificaciones, así el año escolar del ciclo sierra y amazonía terminó de manera no presencial con el "Plan educativo aprendamos juntos en casa" en el marco del Plan educativo Covid-19, mismo que cuenta con una serie de guías y recursos educativos disponibles en el portal web del Ministerio de Educación. Se trata de fichas pedagógicas semanales para el desarrollo en casa por parte de los estudiantes y de un "acompañante" (Ministerio de Educación, 2020). Están organizadas a manera de proyectos que integran diferentes asignaturas y por subniveles educativos de la educación Básica (elemental, media o superior). Cada subnivel de Educación General Básica, -EGB- incluye tres años de escolaridad. Llama ampliamente la atención el divorcio que se encuentra entre estos documentos microcurriculares y currículo nacional de los niveles de educación obligatoria, este último es un currículo por destrezas con criterios de desempeño. Las guías educativas parten de objetivos de aprendizaje semanales que no explicitan las destrezas curriculares ni los valores del perfil de salida de la educación obligatoria.

Ante la imposibilidad de llegar con procesos de educación virtual a todos los niños y jóvenes del sistema educativo se prevén otras medidas como la incorporación de franjas educativas en canales de televisión y radios comunitarias, en diferentes horarios dependiendo el nivel educativo de los estudiantes. En TV se trató del programa “A-prender 
la tele" del Ministerio de Educación. Así mismo, el Ministerio de Educación imprimió guías didácticas para su entrega a estudiantes que tienen imposibilidad de conexión de internet para la continuidad de los procesos educativos.

Hasta aquí hemos referido a las políticas curriculares de parte del Ministerio de Educación, tanto sobre las características de su contenido, como de las formas de implementación del mismo. Para la comprensión sobre las concreciones curriculares de nivel micro, trabajamos a partir del concepto de currículo operativo, currículum oficial o currículo real o vivido, según insiste Stenhouse, el estudio del currículum, implica sus dos acepciones: intención y realidad (Stenhouse, 1991, p. 27). Esta comprensión tendrá mucho que ver, con el ejercicio de la práctica de la docencia, en el trabajo de aula. En condiciones de aislamiento y teletrabajo por la pandemia las prácticas de implementación del currículo van tomando particularidades en relación a los contextos, recursos y niveles educativos.

Estas políticas curriculares a las que hemos referido, al ser de tipo prescriptivas, estarían direccionadas a instrumentalizar los procesos educativos, se caracterizan por ser de tipo regulatorias y homogeneizadoras, en un momento donde la flexibilidad, contextualización y pertinencia tendrían que ser las principales características del diseño curricular y su operativización. En este sentido, se vuelve cada vez más evidente que las orientaciones que se emanan desde el currículo direccionan un ejercicio de la docencia también de tipo instrumental, el profesorado desde esta perspectiva se convierte, en el mejor de los casos en quien aplica un currículo de tipo cerrado.

Lo expuesto abre nuevamente el debate sobre la función social de la escuela y su currículo. Discurre nuevamente la comprensión de currículo entre una concepción tradicional, eficientista, instrumental Tyler (1973), Bobbitt (1918) y las posturas críticas Bourdieu \& Passeron (1979), Freire (1970), Apple (1995), entre otros) para quienes "lo importante no es desarrollar técnicas de cómo hacer el currículo, sino desarrollar conceptos que nos permitan comprender lo que el currículo hace" (Da Silva, 1992, p. 13).

\section{METODOLOGÍA}

Para la consecución de los objetivos planteados en el presente estudio ${ }^{2}$ optamos por enfoques mixtos buscando una triangulación metodológica que combinó el análisis documental, ciberetnografía y encuesta, acudiendo así a métodos cuantitativos, en el caso de la encuesta y cualitativos en el caso de la ciberetnografía que se realizó a través de apoyos virtuales, con el fin de obtener información desde diferentes perspectivas para dotar al estudio de una comprensión más amplia del fenómeno.

Así, el primer procedimiento se enmarca en la revisión pormenorizada de fuentes secundarias (decretos, declaraciones y comunicados ministeriales, reportajes y notas periodísticas) que daban cuenta de las acciones, lineamientos y políticas que el gobierno nacional y su cartera de Estado iban tomando ante la situación, dinámica de la pandemia y sus efectos en el proceso educativo.

Este ejercicio de revisión secundaria devino en la elaboración de una línea de tiempo que permitió identificar con claridad, las decisiones que se tomaban, algunas con un

Este estudio es parte del proyecto de investigación "Educación y docencia en tiempo de emergencia sanitaria por COVID 19" que lo lleva adelante el GIEI (Grupo de Investigación Educación e Interculturalidad de la Universidad Politécnica Salesiana). 
adecuado acierto, pero otras, daban cuenta del cierto grado de improvisación e incluso, de desconocimiento de la realidad ecuatoriana.

El segundo procedimiento empleado se inscribió en la tradición cualitativa; acudimos a la aplicación de una variante de la etnografía tradicional, es decir, la etnografía digital (Pink et al., 2016, p. 115). Partiendo del supuesto que todo cuanto se produce en las interacciones digitales, de alguna manera son el reflejo de las acciones materiales de la realidad presencial. El contexto de emergencia actual nos trasladó a no tan nuevos, pero aún poco explorados, espacios de interacción y socialización. Gran parte de nuestro tiempo y las actividades que desarrollamos cotidianamente, sobre todo desde el inicio de la pandemia, se centran en las pantallas de los dispositivos tecnológicos. Las actividades docentes no están al margen de ello. Por eso, la aplicación de métodos que procuran adaptarse a estas 'nuevas normalidades' y realidades se tornan imprescindibles en el campo investigativo. En ese sentido, optamos por realizar un ejercicio etnográfico digital, una observación no participante del proceso de aprendizaje, identificando las "virtudes" y "dificultades" que se produjeron durante el desarrollo de las actividades pedagógicas.

El tercer procedimiento, de corte cuantitativo, fue la implementación de una encuesta a docentes. El instrumento se aplicó de forma digital a través de la herramienta formularios de Google Forms. La encuesta se aplicó a profesores del sistema educativo ecuatoriano de las regiones Sierra y Amazonía. Por encontrarnos en una situación de emergencia sanitaria, recurrimos a las redes de contactos que el Grupo de Investigación Educación e Interculturalidad, -GIEI- de la Universidad Politécnica Salesiana del Ecuador dispone. Por esto, el tipo de muestreo seleccionado para el presente estudio fue el aleatorio estratificado, entendiendo que la muestra incluye subgrupos o estratos que conforman la población objetivo [...] subgrupos o unidades de análisis que difieren las características entre sí (Otzen y Manterola, 2017, p. 35). En el caso del presente estudio los estratos corresponden al género, localidad de trabajo, tipos de sostenimiento institucional, edad y formación profesional de los docentes.

Asimismo, la decisión de aplicar el instrumento en las dos regiones respondió a los criterios muestrales de permanencia y transición de la actividad docente, es decir, las instituciones educativas se encontraban en pleno desarrollo del segundo quimestre del año lectivo, por lo tanto, los docentes permanecían con actividades pedagógicas y a su vez, por la emergencia sanitaria, la modalidad de estudios transitó a modalidad en línea y de teletrabajo.

\section{ANÁLISIS Y RESULTADOS}

La función primordial de todo Estado es garantizar el bienestar de sus ciudadanos. Para cumplir este objetivo las orientaciones jurídicas se constituyen en el marco normativo que organiza la vida social de un país. En el caso ecuatoriano, la Constitución de 2008 en su artículo 26 se asegura que "la educación es un derecho de las personas a lo largo de la vida y un deber ineludible e inexcusable del Estado (Asamblea Nacional del Ecuador, 2008) [...]": Ante la situación de emergencia sanitaria de orden global el gobierno nacional intentó cumplir y asegurar el mencionado derecho, sin embargo, las directrices de políticas educativas que emergieron a medida que la crisis avanzaba no fueron siempre las acordes al enunciado constitucional, a saber que las condiciones de los educandos no se encontraban plenamente aseguradas con anterioridad, porque los recortes presupuestarios en el sector 
educativo han sido una constante en los últimos 3 años de gobierno nacional (Observatorio Social del Ecuador, 2019).

Cuando hablamos de políticas sociales y de forma más específica de políticas educativas y curriculares podemos mencionar que es necesario considerar cuatro elementos centrales que permiten identificar la existencia de una política: La primera, implicación del gobierno, en el caso que nos ocupa podemos entrever que el gobierno a través de su cartera de estado intervino con acciones puntuales como la suspensión de actividades escolares, elaboración del Plan Educativo Covid-19 y más adelante el Programa Educa Contigo. Empero de estas directrices, ciertamente necesarias, consideramos que la implicación gubernamental en el tema educativo quedó como una tarea inconclusa porque si bien el tema de la pandemia fue una situación emergente, tampoco fue algo que nos llegó de forma inesperada. Las previsiones de instituciones intergubernamentales, sobre todo del sistema de Naciones Unidas en el mes de febrero, ya daban cuenta que la enfermedad se propagaba con rapidez por Europa luego de haber confinado a dos ciudades de China y que pronto estaría en América.

Estas advertencias realizadas por expertos en salud fueron poco o nada consideradas por el gobierno nacional; esta afirmación se sustenta en la escasa o mejor dicho nula, previsión y provisión de recursos tecnológicos que se entregaron a docentes y estudiantes. En la misma dirección, no se realizó un diagnóstico a los docentes con la finalidad de identificar las competencias digitales que este sector poseía, sin embargo, las directrices apuntaron a la implementación de teleducación sin considerar que los docentes carecían de formación y capacitación previa. En suma, la implicación del gobierno ante un posible problema no se efectuó.

El segundo elemento es la identificación de problemas. Como ya lo mencionamos líneas atrás, el problema del virus y sus consecuencias no fueron temas ajenos para el gobierno y la ciudadanía ecuatoriana, durante los meses de enero y primeros días de febrero los noticieros y portadas de periódicos otorgaban como tema central al coronavirus y sus fatales secuelas en Asia y Europa. A pesar de ello, en Ecuador, el problema no fue tratado y las posibles acciones educativas no fueron debidamente planificadas a pesar de que el problema era inminente.

Un tercer factor tiene que ver con las definiciones de objetivos que debe tener una política y en el caso educativo, los objetivos de las políticas se deben plasmar a partir de los principios postulados en la Constitución del Ecuador. En ese sentido, las políticas propuestas por el gobierno del Presidente Moreno no se alinearon con lo establecido y normado en la Carta Magna. El resultado de este "desliz político" que infringe la norma es el centenar e incluso miles de niñas, niños y adolescentes que abandonaron sus estudios, $u$ otros que seguramente no continuarán su proceso. Según una nota periodística se reporta que el Estado perdió el rastro de 17700 estudiantes del régimen sierra y amazonía, y de 5600 del régimen costa (Diario EL Comercio, 2020). Estos datos dan cuenta de la magnitud del problema provocado por ausencia de diagnósticos, planificación y sobre todo de implementación de políticas públicas que garanticen las normas constitucionales establecidas.

Finalmente, un cuarto elemento está relacionado con el proceso, el cual se encuentra destinado a cambiar una situación problemática (Roth, 2009, p. 27), que por el contrario de cambiarse o al menos, modificarse, como se demuestra con los datos de deserción escolar, expuestos anteriormente y sobre todo las narrativas de los docentes, se afianzó y amplificó.

Ahora bien, entrando en materia cuantitativa, la totalidad de respuestas válidas del presente trabajo fue de 606 docentes de los cuales el $68 \%$ corresponden al género femenino, el $34 \%$ se autoidentifican como masculino, el $1 \%$ como perteneciente a la comunidad lgbti y un $1 \%$ se autoidentifican como mixto. 
Figura 1. Docentes por género.

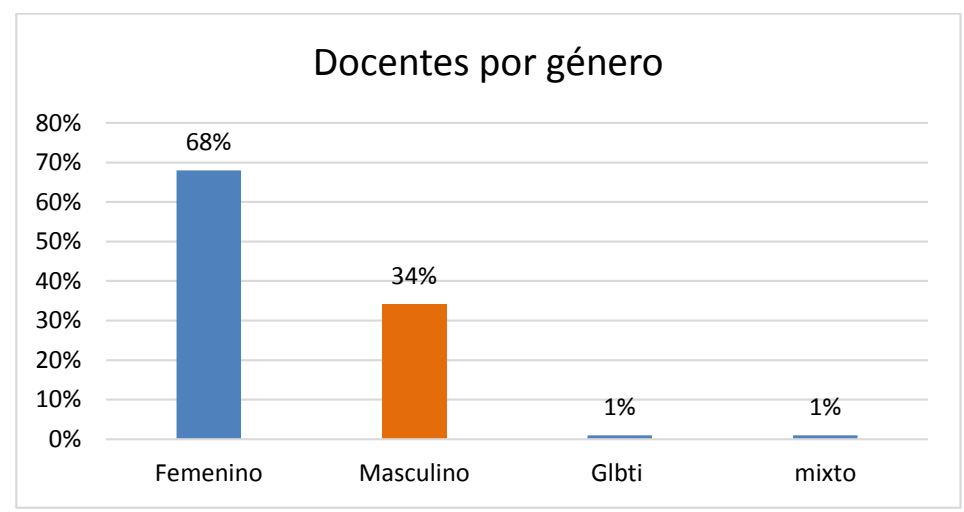

Fuente: Encuesta virtual realizada a docentes ecuatorianos, 2020.

Es claro que el porcentaje mayoritario se inclina hacia el género femenino por un asunto copiosamente trabajado por la literatura educativa, nos referimos a la feminización de la labor docente. En el presente estudio, se ratifica que la presencia de las mujeres en los espacios escolares continúa prevaleciendo y con más acento, en los niveles iniciales de escolaridad.

En lo que respecta a las edades de los encuestados el $39 \%$ de los docentes se encuentran en la franja de 30 a 40 años de edad. El 25\% se ubican entre los 40 y 50 años, seguido de un $20 \%$ de 50 a 60 años. Los docentes más jóvenes, de 20 a 30 años, corresponden al $16 \%$ y finalmente, se ubican los docentes de 60 a 70 años con $1 \%$.

Figura 2. Docentes por franja etaria.

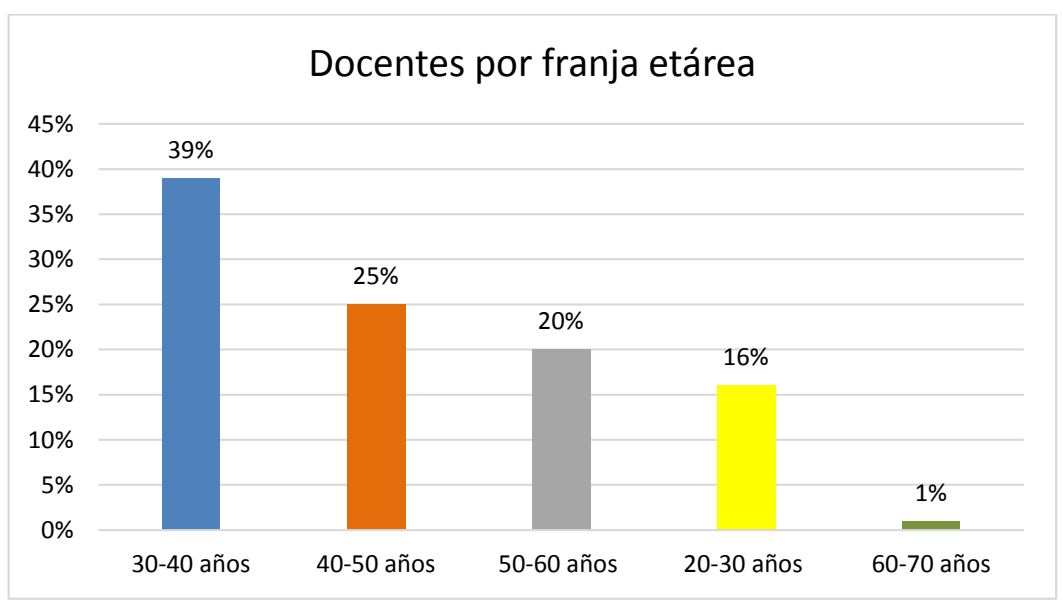

Fuente: Encuesta virtual realizada a docentes ecuatorianos, 2020. 
Estos datos nos parecen altamente significativos porque nos permite evidenciar, al menos en la muestra del estudio, que la población docente que enfrentó los primeros momentos de la pandemia, se encuentra conformada por un segmento poblacional etario que ha tenido cercanías al uso de tecnologías. Si sumamos los rangos de edad de 20 a 50 años obtenemos que el $79 \%$ de los docentes se encuentran en estas edades. Suponemos que la edad de los docentes se constituye en un factor diferenciador a la hora de cambiar y modificar la práctica docente a un modelo de teleeducación.

Los datos acerca del sostenimiento institucional reflejan claramente que el perfil de los docentes que colaboraron en el presente estudio centra su labor en instituciones públicas o fiscales $43 \%$. Los docentes que laboran en instituciones particulares o privadas corresponden al $31 \%$ y finalmente un $26 \%$ son docentes que cumplen su labor en centros educativos fiscomisionales.

Figura 3. Docentes por sostenimiento Institucional.

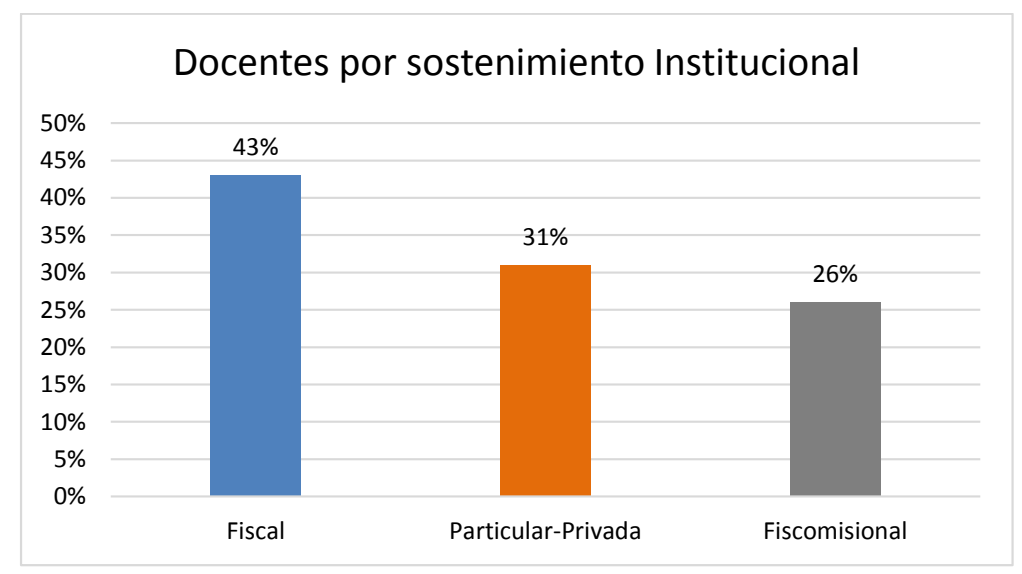

Fuente: Encuesta virtual realizada a docentes ecuatorianos, 2020.

En el artículo 37 de la Ley Orgánica de Educación Intercultural, -LOEI- se establece la composición del sistema de educación nacional. En el mismo artículo se menciona la desconcentración del sistema de educación intercultural bilingüe, es decir, en el caso ecuatoriano encontramos dos sistemas: el hispano y el sistema de educación intercultural bilingüe. En la encuesta, el $71 \%$ de los docentes prestan sus servicios en instituciones educativas que son parte del sistema hispano. El 29\% pertenecen al sistema de educación intercultural bilingüe, finalmente, e1\% no proporciona una respuesta a esta pregunta. 
Figura 4. Docentes por tipo de institución Educativa según jurisdicción.

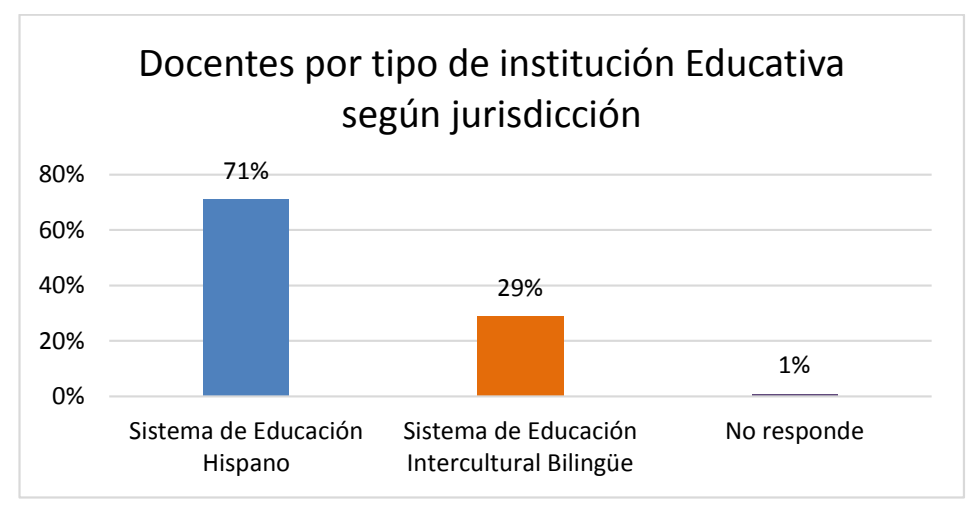

Fuente: Encuesta virtual realizada a docentes ecuatorianos, 2020.

Los resultados de la encuesta en lo que refiere a las concreciones curriculares, siendo este de tipo prescriptivo, muestran una amplia aplicación del currículo Plan Covid-19 en las respuestas de los docentes encuestados.

Figura 5. Docentes que aplica el Plan Covid-19.

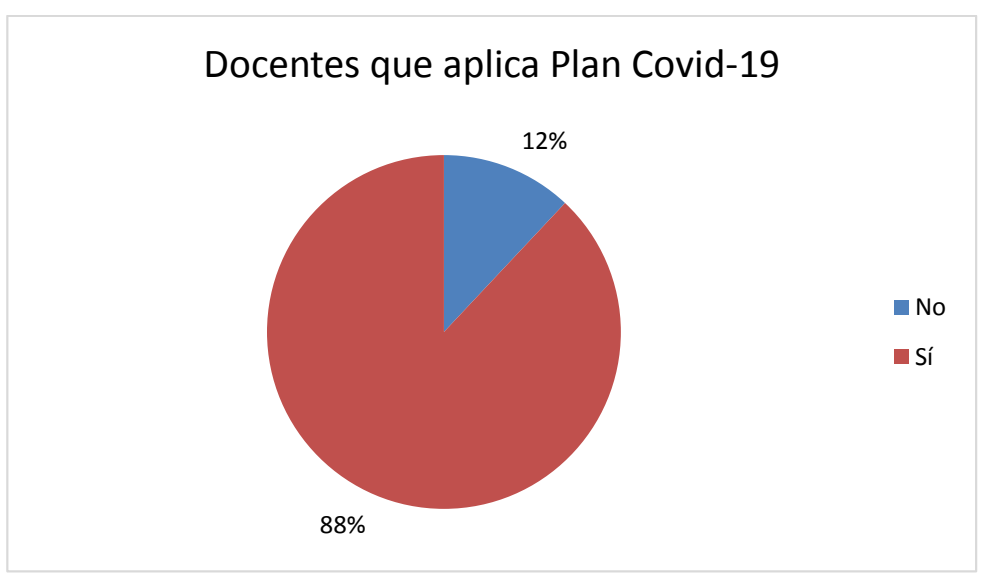

Fuente: Encuesta virtual realizada a docentes ecuatorianos, 2020.

Ahora, vale la pena mencionar que, si bien la mayor parte de docentes $(88 \%)$ afirman aplicar el Plan Covid-19, en otra pregunta de tipo abierta donde se interroga a los docentes cuáles son las mejores actividades para el aprendizaje, un número muy limitado de profesores refiere a las del Plan Covid-19 como las más efectivas, tal como muestra la siguiente gráfica. Asumimos que este comportamiento disímil en los datos obedece a la 
lógica de cumplimiento marcada en la práctica profesional, es decir, al constituirse la aplicación del Plan Covid-19 como un mandato ministerial los docentes optan por asumir esta directriz, sin embargo, en la operatividad del desarrollo pedagógico, la dinámica y las propias realidades conducen a los docentes a la aplicación de otras actividades.

Figura 6. Mejores actividades de aprendizaje.

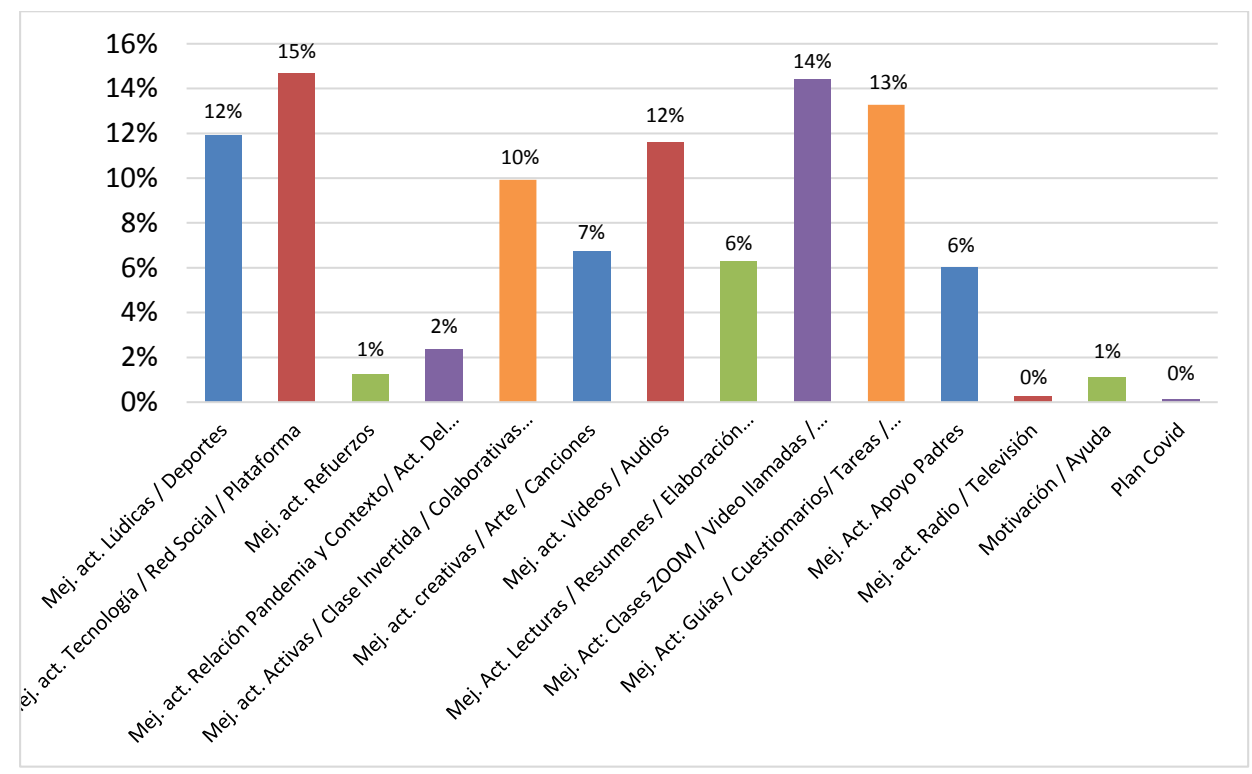

Fuente: Encuesta virtual realizada a docentes ecuatorianos, 2020.

En concordancia con lo anterior, la data muestra cómo los docentes, casi en su totalidad realizan una priorización de los contenidos curriculares (principalmente destrezas) para el trabajo en la cotidianidad en el aula o realizan un total rediseño respecto al currículo previo a la pandemia. Así mismo, un importante porcentaje de docentes dice incluir temas de contexto en su planificación de aula.

Lo expuesto en estas dos gráficas muestra que, si bien por un lado el carácter prescriptivo del currículo y su carácter regulador exige de la docencia su observación, sin embargo, los hallazgos que arroja la gráfica 6 presentan una realidad mucho más compleja en vista del enorme abanico de estrategias que planifican los docentes para los procesos de enseñanza-aprendizaje. Las guías del plan Covid-19 son apenas una de las opciones y entre ellas las de menor referencia docente y como se corrobora más adelante con los datos de la cyberetnografía, son incluso de tipo complementaria, dando lugar a una interpretación relevante entre aquello que prescribe la política curricular y lo que la vida escolar en tiempo de pandemia y por teletrabajo exige. 
Figura 7. Planificación de Aula

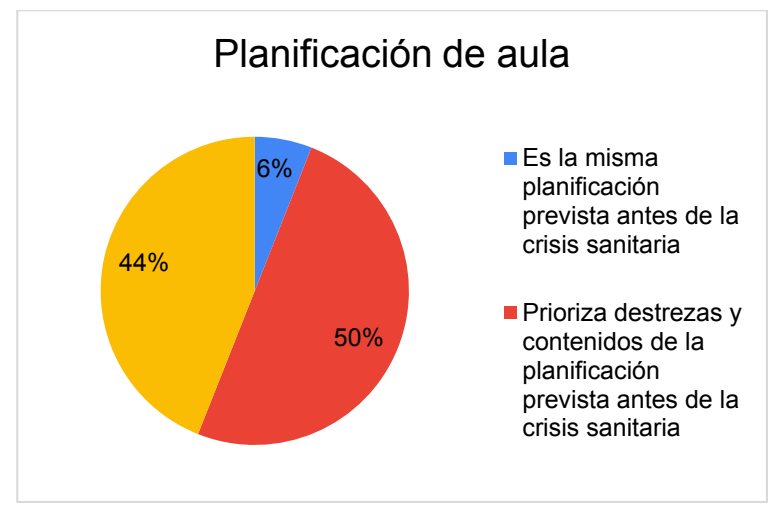

Fuente: Encuesta virtual realizada a docentes ecuatorianos, 2020.

Figura 8. Inclusión temas de contexto en la planificación de aula.

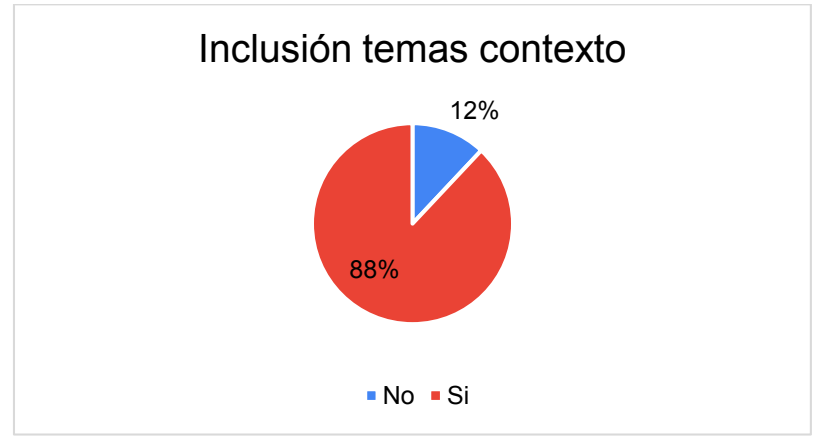

Fuente: Encuesta virtual realizada a docentes ecuatorianos, 2020.

Los datos de la encuesta fueron contrastados con la cyberetnografía de las concreciones microcurriculares en varios grados de educación básica en 20 instituciones educativas públicas, privadas y fiscomisionales, realizadas por los estudiantes de la carrera de formación docente de Educación Básica de la UPS, entre los meses de mayo y junio de 2020, en la asignatura Cátedra Integradora IV: Modelos curriculares.

Algunos datos relevantes sobre las concreciones curriculares en este período, obtenidos de los registros de observación fueron:

En la institución Fiscal se utiliza de manera permanente en todas las clases observadas el plan educativo Covid-19, mientras que en la escuela privada y el colegio municipal se dio prioridad al plan anual con sus destrezas y objetivos iniciales; pero a su vez se utilizaron valores, el diálogo y procesos de escucha para la comprensión de la 
pandemia actual y el estado de ánimo de sus educandos (Andrade \& Espín, 2020, Registro de observación).

Las actividades de aprendizaje en las instituciones públicas están establecidas según en el plan Covid-19 junto con la realización de las actividades del libro del ministerio de educación y proyectos las actividades realizadas por los estudiantes son las investigaciones, lecturas de libros, resolver cuestionarios, consultas etc. Mientras que en las instituciones privadas utilizan sus propias planificaciones junto con las destrezas que se desea lograr cada semana junto con la utilización de actividades del texto Santillana las actividades realizadas por los estudiantes son realizar experimentos, consultas, observar videos, lecturas de libros, resolver cuestionarios, etc (Pinza \& Caiza, Registro de observación, 2020).

Los temas a trabajar correspondían a los contenidos propuestos en el libro, para este último mes de trabajo se tanto los contenidos de la última unidad, la Unidad 6 de los libros de texto de Santilla. En inglés el tema central de esta Unidad 6 es Passive Voice tenses, en Matemáticas la Unidad 6 abarcaba el tema de Estadística y Probabilidad, Ciencias Sociales trabajo la unidad de Ciudadanía, Derechos y Deberes y finalmente Ciencias Naturales trato la unidad de Biodiversidad y el impacto del ser humano sobre la naturaleza (Gualichico, Flores, \& Orquera, 2020, Registro de observación).

En cuanto al Plan COVID-19, los estudiantes 1 y 2 de la escuela fiscal sí lo utilizaban, semanalmente la docente envía un PDF con un proyecto escolar nuevo, acompañado de su respectivo objetivo de aprendizaje, instrucciones, y actividades. Por otra parte, los docentes de los estudiantes 3 y 4 (escuelas particulares) mantenían una metodología didáctica, adaptada a la emergencia sanitaria que vive el país, garantizando así una formación virtual de calidad y calidez, trabajando paralelamente en la contención emocional de los estudiantes (Santillán \& Bonilla, 2020, Registro de observación).

Las observaciones realizadas en las escuelas dan cuenta de las diferencias en la aplicación del Plan Educativo Covid-19 en las distintas instituciones, en relación con su sostenibilidad y contexto en que se produce el acto educativo. De allí que, retomando a Stenhouse, habrá que diferenciar entre la intencionalidad del currículo y la realidad. Pero, por otro lado, también hay que tener en cuenta la funcionalidad del documento curricular considerando la diversidad de los sujetos y sus contextos.

Así mismo, se evidencia que, en gran parte de las instituciones educativas, durante el confinamiento por la pandemia, se mantiene el libro de texto como uno de los principales recursos. Los proyectos educativos que hacen parte del Plan educativo Covid-19, se utilizan en varias escuelas de manera complementaria, tal como se evidencia en los registros de la observación realizada:

Los recursos que utiliza la maestra son el texto del Ministerio de Educación que fueron entregado al principio del año, allí realizan actividades con respecto al tema a tratar, guías y planificación de tareas y actividades enviadas por semana y de diferentes áreas. Hace uso de lecturas del Plan Covid-19 dentro de las planificaciones y las actividades que están establecidas en ese documento para que los estudiantes realicen según la semana correspondiente, además de proyectos y talleres, y así mismo deben realizar el portafolio estudiantil que será entregado en la institución educativa y será evaluado por docentes (Gaona, Casagualpa, \& Yánez, 2020, Registro de observación). 
En suma, se puede observar que, una vez suspendido el trabajo educativo presencial por la pandemia, la actividad educativa se vio abocada a llevarse a cabo de diversas formas, dependiendo de los contextos, accesos y conectividad. Así mismo el tipo de actividades de aprendizaje y los recursos educativos que se realizaron en el proceso de enseñanzaaprendizaje dependerían de las diferentes realidades.

La siguiente gráfica muestra la respuesta de los docentes cuando se les pregunta cuáles son los recursos educativos más utilizados. Dado que la encuesta fue contestada por docentes con acceso a internet en más de un $91 \%$, al ser este el canal por el que se envió el formulario, la mayor parte de ellos, también señalan que, entre los recursos más utilizados para su trabajo, se encuentran las plataformas educativas digitales. Sin embargo, y en concordancia con lo observado en las prácticas, los textos escolares, físicos o digitales siguen siendo un importante recurso educativo, principalmente para el envío de tareas con un $42 \%$ de docentes que indica enviar como tarea el resolver las actividades del texto. Según se observa en las siguientes gráficas.

Por el contrario, un número muy reducido de docentes manifiesta utilizar la radio o la televisión, de allí que es importante analizar en futuras investigaciones la efectividad del programa "A-prender la tele", como parte de las estrategias del Ministerio de Educación para lograr el acceso de los estudiantes a la educación durante el tiempo de pandemia.

Figura 9. Recursos educativos más utilizados por los docentes.

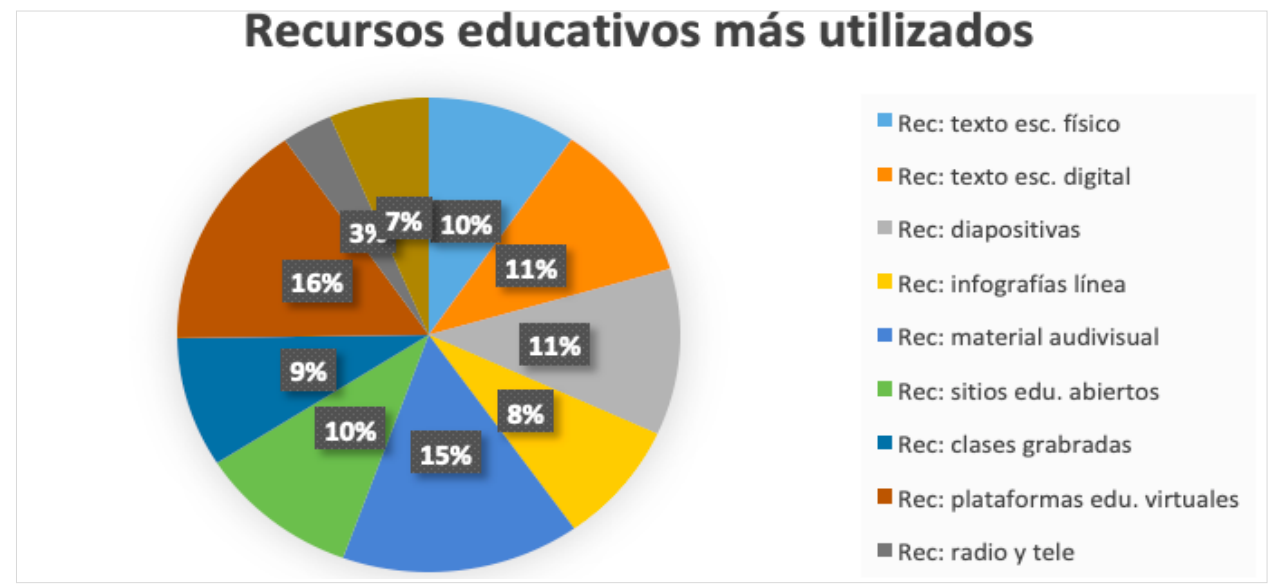

Fuente: Encuesta virtual realizada a docentes ecuatorianos, 2020. 
Figura 10. Priorización del tipo de tareas.

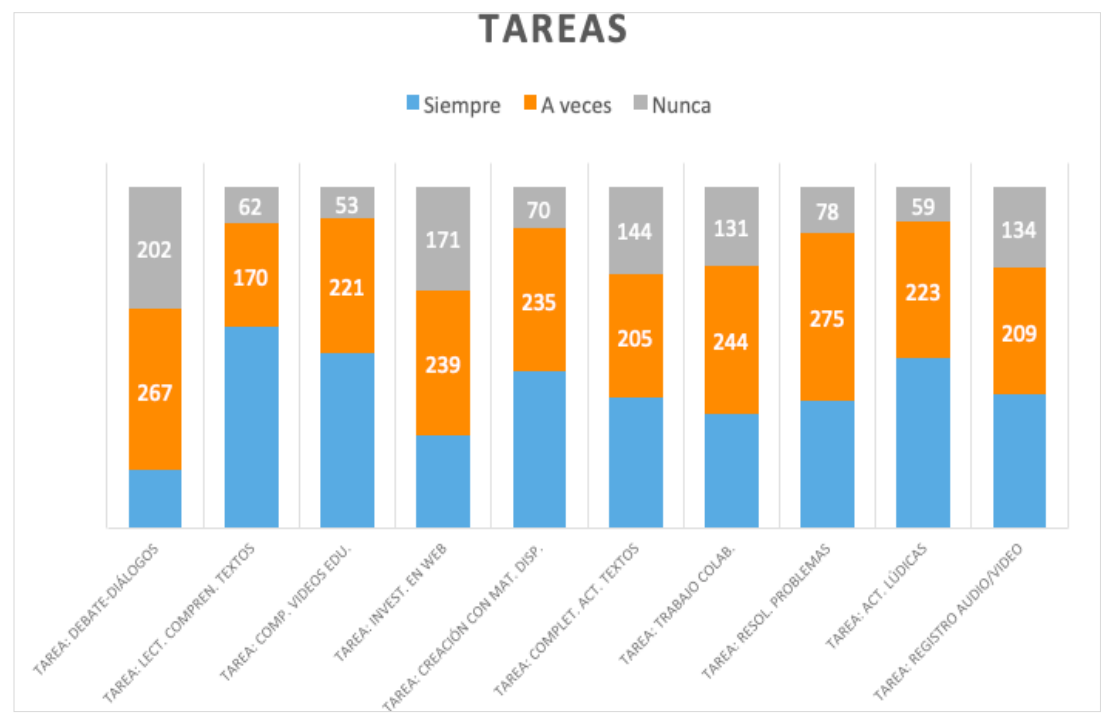

Fuente: Encuesta virtual realizada a docentes ecuatorianos, 2020.

Para los estudiantes que no tienen conectividad, se previó la entrega de fichas pedagógicas semanales a los estudiantes que deberán ser trabajadas con el apoyo de un acompañante. En uno de los contextos observados, el registro muestra expresa lo siguiente:

Debido a las condiciones socio-económicas bajas del niño observado, el acompañamiento del padre del niño se limita a la revisión de tareas por la falta de tiempo, ya que sobreviven al día, mientras en la realización el apoyo es realizado por el hermano mayor y un vecino que le facilita la comprensión de las fichas y le aporta ideas para el trabajo (Cedeño, Cuaspud, \& Maldonado, 2020, Registro de observación).

Sin embargo, de las dificultades que presenta la falta de conectividad o las distintas condiciones contextuales de los educandos, es importante destacar el trabajo y esfuerzo docente, tal como se concluye en los trabajos de observación.

En todo el proceso de observación se evidenció como los docentes de manera asincrónica se comunicaban con los estudiantes por diversas plataformas, en algunos casos había horarios específicos y en otros no, sin embargo, el docente estaba dispuesto a solucionar las inquietudes de los estudiantes para de este modo ayudarlos a continuar con su proceso de aprendizaje (Cedeño, Cuaspud, Maldonado, 2020, Registro de observación).

Los registros de lo observado y la postura pública de muchos docentes en diferentes medios de comunicación dan cuenta de una docencia crítica; la limitada aplicación de las 
guías del plan Covid y sus adaptaciones muestran también la crítica a un diseño curricular poco consensuado con los docentes quienes al fin y al cabo llevan adelante la tarea educativa, pero a quiénes no se les ha consultado, ni sobre el currículo, ni sobre las necesidades que ellos pueden tener para desarrollar su trabajo; pero que sin embargo de ello han sostenido todos estos meses la educación de los niños/as y jóvenes.

\section{CONSIDERACIONES FINALES}

Un currículo no es un sumario, o una lista de contenidos, corresponde por el contrario a las decisiones sobre la enseñanza; que se enseña, cómo se enseña y los recursos que se dispone y se requiere. Se define desde las necesidades educativas de los educandos y sus contextos, por tanto, un currículo para una situación de emergencia debe brindar las orientaciones educativas pertinentes a las distintas realidades donde este se concrete. Nos enfrentamos entonces frente a la necesidad de pensar que el currículo es siempre vivo y dinámico. Junto con ello, se requiere poner a disposición todos los medios y herramientas que permitan su ejecución eficiente, efectiva y eficaz.

Las decisiones educativas, sobre el diseño y desarrollo curricular deberán ser siempre consensuadas con los diferentes actores de la comunidad educativa. El diseño curricular es participativo y crítico y en su elaboración deberán implicarse los docentes. "No hay desarrollo de un currículum sin el desarrollo del profesor". Por tanto, la implicancia docente en el diseño y desarrollo de las políticas educativas y curriculares será siempre de fundamental consideración. Así mismo, la docencia deberá ser vista como una práctica reflexiva. El profesorado es más que un ejecutor de un currículo, su práctica será siempre reflexiva.

Queda claro que cualquier política curricular no tienen un carácter neutral. Pero por otro lado no hay que perder de vista que en su concreción se pone en juego la enorme capacidad de agencia del profesorado ecuatoriano

Por otro lado, en el contexto de la pandemia se han reflejado las improvisaciones por parte del Ministerio de Educación con la implementación del Plan Educativo Covid-19 y los programas de radio y televisión que son insuficientes ante la diversidad de contextos en los que se encuentran los estudiantes y sus familias. Es un tema de preocupación generalizada la escasez de accesos a recursos tecnológicos y conectividad al servicio de internet, imposibilitando sus aprendizajes de forma sincrónica.

En ese sentido, es importante considerar los datos expuestos de deserción escolar, formas de aprendizaje, pertinencia de las prácticas curriculares y la situación de los docentes en el contexto de la pandemia y seguramente tendremos que pensar el escenario de post pandemia a través de políticas de Estado, permanentes y que, sobre todo, garanticen los derechos consagrados en los instrumentos jurídicos del país.

Es importante señalar que la pandemia desnudó y a la vez profundizó las desigualdades educativas, por ello, se torna imprescindible fortalecer las inversiones presupuestarias en el sector educativo en dos campos: capacitación docente en competencias digitales y por supuesto la ampliación de cobertura de redes de internet. Es claro que el compromiso de los docentes se encuentra presente e intacto, pero estamos seguros de que con mejores condiciones materiales la labor de los profesores sería mejor de lo que ya tenemos.

El escenario post pandemia es incierto, no sabemos cuándo llegará, ni cómo será, pero la crisis global deja por sentado la vulnerabilidad de los seres humanos y las improvisaciones 
gubernamentales en varios sectores, pero de forma indiscutible en el educativo a la hora de gestionar una situación como la vivida durante este período; las enseñanzas sin duda son múltiples y debemos recogerlas para afianzar y mejorar nuestra labor docente.

Finalmente, durante el tiempo de pandemia la producción de eventos académicos ha sido prolífica; las conferencias y debates alrededor de las problemáticas educativas ocuparon y ocupan un lugar preponderante en diferentes escenarios, sin embargo, consideramos que la pertinencia de analizar las respuestas políticas, traducidas en acciones curriculares, que los gobiernos de los países latinoamericanos otorgan se torna necesario profundizar.

\section{REFERENCIAS BIBLIOGRÁFICAS}

Apple, M. (1995). Education and power. Routledge.

Asamblea Nacional del Ecuador. (2008). Constitución de la República del Ecuador 2008. http://bit. $1 \mathrm{y} / 343 \mathrm{suPg}$

Bobbitt, F. (1918). El currículo. The Riverside Press Cambridge.

Bourdieu, P. \& Passeron, J. C. (1979). La reproducción. Editorial Laia S.A.

Capella, J. (n.d.). Políticas educativas. In S.F. https://bit.ly/2Je5k30

Da Silva, T. (1992). O que produz e o que reproduz em educacao. Editora Artes Médicas Sul.

Diario El Comercio. (2020). Abandono escolar en la pandemia. https://bit.ly/2GxmDuT

Freire, P. (1970). Pedagogía del oprimido. Siglo XXI Argentina Editores, S. A.

Ministerio de Educación del Ecuador. (2020). La Educación a través de plataformas digitales. La Educación a Través de Plataformas Digitales. https://bit.ly/2VZ9O07 . (2020). Plan Educativo Covid-19. https://educacion.gob.ec/

Observatorio Social del Ecuador, O. (2019). Situación de la niñez y adolescencia en Ecuador/ Una mirada a través de los ODS. https://bit.ly/2HWxBuk

Otzen, T. \& Manterola, C. (2017). Técnicas de Muestreo sobre una Población a Estudio. Int. J. Morphol, 35(1), 227-232. http://bit.ly/2BYprvm

Oviedo, A. (2020). La educación en tiempos del COVID-19. La Educación En Tiempos Del COVID-19. https://bit.ly/3bhzD14

Pink, S., Horst, H., Postill, J., Hjorth, L., Lewis, T. \& Tacchi, J. (2016). Digital Ethnography. Principles and Practice. Sage.

Pulido, O. (2017). Política pública y política educativa: una reflexión sobre el contexto. Educación y Ciudad, 33, 13-28. https://bit.ly/2GgVaOa

Roth, A. (2009). Políticas Públicas, Formulación, Implementación y Evaluación. Ediciones Aurora. https://bit.ly/30HiOtY

Stenhouse, L. (1991). Investigación y desarrollo del curriculum. Ediciones Morata.

Tyler, R. (1973). Principios básicos del currículo. Troquel. https://bit.ly/2TE2TZF

Velásquez-Gavilanes, R. (2009). Hacia una nueva definición del concepto "política pública" Desafíos, 20, 149-187. https://bit.ly/3jD3S7A 